\title{
Kybernetika
}

\section{Vlasta Kaňková}

Stability, empirical estimates and scenario generation in stochastic optimization applications in finance

Kybernetika, Vol. 53 (2017), No. 6, 1026-1046

Persistent URL: http://dml.cz/dmlcz/147083

\section{Terms of use:}

(C) Institute of Information Theory and Automation AS CR, 2017

Institute of Mathematics of the Czech Academy of Sciences provides access to digitized documents strictly for personal use. Each copy of any part of this document must contain these Terms of use.

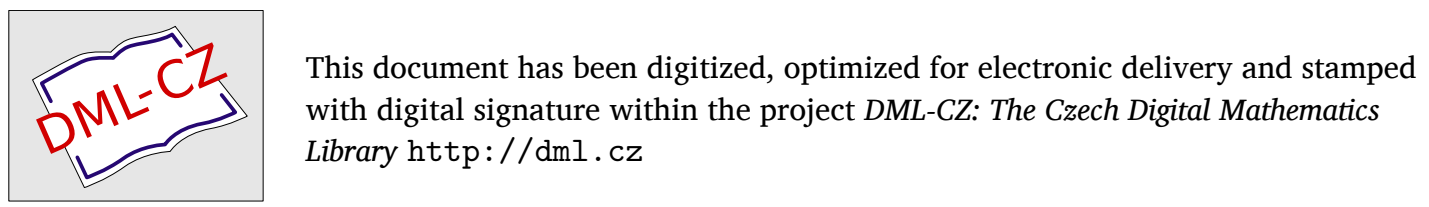




\title{
STABILITY, EMPIRICAL ESTIMATES AND SCENARIO GENERATION IN STOCHASTIC OPTIMIZATION - APPLICATIONS IN FINANCE
}

\author{
VLASTA KAŇKOVÁ
}

Economic and financial processes are mostly simultaneously influenced by a random factor and a decision parameter. While the random factor can be hardly influenced, the decision parameter can be usually determined by a deterministic optimization problem depending on a corresponding probability measure. However, in applications the "underlying" probability measure is often a little different, replaced by empirical one determined on the base of data or even (for numerical reason) replaced by simpler (mostly discrete) one. Consequently, real one and approximate one correspond to applications. In the paper we try to investigate their relationship. To this end we employ the results on stability based on the Wasserstein metric and $\mathcal{L}_{1}$ norm, their applications to empirical estimates and scenario generation. Moreover, we apply the achieved new results to simple financial applications. The corresponding model will a problem of stochastic programming.

Keywords: stochastic programming problems, probability constraints, stochastic dominance, stability, Wasserstein metric, $\mathcal{L}_{1}$ norm, Lipschitz property, empirical estimates, scenario, error approximation, financial applications, loan, debtor, installments, mortgage, bank

Classification: $90 \mathrm{C} 15$

\section{INTRODUCTION}

Let $(\Omega, \mathcal{S}, P)$ be a probability space; $\xi\left(:=\xi(\omega)=\left(\xi_{1}(\omega), \ldots, \xi_{s}(\omega)\right)\right.$ an $s$-dimensional random vector defined on $(\Omega, \mathcal{S}, P) ; F\left(:=F_{\xi}(z), z \in \mathbb{R}^{s}\right)$ the distribution function of $\xi ; P_{F}, Z_{F}$ the probability measure and a support corresponding to $F$. Let, moreover, $g_{0}\left(:=g_{0}(x, z)\right)$ be a real-valued function defined on $\mathbb{R}^{n} \times \mathbb{R}^{s} ; X_{F} \subset X \subset \mathbb{R}^{n}$ a nonempty set generally depending on $F, X \subset \mathbb{R}^{n}$ a nonempty "deterministic" set. If $\mathrm{E}_{F}$ denotes the operator of mathematical expectation corresponding to $F$ and if for $x \in X$ there exists a finite $\mathrm{E}_{F} g_{0}(x, \xi)$, then one-stage (static) "classical" stochastic optimization problem can be introduced in the form:

Find

$$
\varphi\left(F, X_{F}\right)=\inf \left\{\mathrm{E}_{F} g_{0}(x, \xi) \mid x \in X_{F}\right\} .
$$

DOI: 10.14736/kyb-2017-6-1026 
To our purpose we recall only special cases of $X_{F}$. We consider the case $X_{F}=X$ ("deterministic" constraints); the case when there exist continuous functions $\bar{g}_{i}\left(:=\bar{g}_{i}(x)\right.$, $\left.x \in \mathbb{R}^{n}\right), i=1, \ldots, s$ and $\bar{g}_{i}(x, z), i=1, \ldots, l$ defined on $\mathbb{R}^{n} \times \mathbb{R}^{s}$ such that the following constraints sets can be defined:

$$
\begin{aligned}
X_{F}\left(:=X_{F}(\alpha)\right)= & \bigcap_{i=1}^{s}\left\{x \in X: P_{F}\left[\omega: \bar{g}_{i}(x) \leq \xi_{i}\right] \geq \alpha_{i}\right\}, \\
& \alpha_{i} \in(0,1), i=1, \ldots, s, \quad \alpha=\left(\alpha_{1}, \ldots, \alpha_{s}\right)
\end{aligned}
$$

known, from the stochastic programming literature, as the problem with individual probability constraints,

$$
X_{F}\left(: X_{F}(\bar{\alpha})\right)=\left\{x \in X: P_{F}\left[\omega: \bar{g}_{i}(x, \xi) \leq 0, i=1, \ldots, l\right] \geq \bar{\alpha}\right\}, \bar{\alpha} \in(0,1),
$$

known as joint probability constraints.

To define the last constraints sets $X_{F}$ let $g(:=g(x, z))$ be a function defined on $\mathbb{R}^{n} \times \mathbb{R}^{s}$, $Y(:=Y(z)):=Y(\xi)$ a random value with the distribution function $F_{Y}$, such that for every $x \in X$ there exist finite $\mathrm{E}_{F} g(x, \xi), \mathrm{E}_{F} Y(\xi)$. Furthermore, let

$$
F_{g(x, \xi)}^{2}(u)=\int_{-\infty}^{u} F_{g(x, \xi)}(y) \mathrm{d} y, \quad F_{Y(\xi)}^{2}(u)=\int_{-\infty}^{u} F_{Y(\xi)}(y) \mathrm{d} y, \quad u \in \mathbb{R}^{1} .
$$

$$
X_{F}=\left\{x \in X: F_{g(x, \xi)}(u) \leq F_{Y(\xi)}(u) \text { for every } u \in \mathbb{R}^{1}\right\}
$$

is known as first order stochastic dominance constraints,

$$
X_{F}=\left\{x \in X: F_{g(x, \xi)}^{2}(u) \leq F_{Y(\xi)}^{2}(u) \text { for every } u \in \mathbb{R}^{1}\right\}
$$

as second order stochastic dominance constraints. (For more information about stochastic dominance see, e.g., [26].)

Evidently, just introduced problems are often rather complicated as from the theoretical so from the numerical point of view. Moreover, employing them to applications, other difficulties can appear. We recall some essential of them:

1. the "underlying" distribution function $F$ can be a little changed;

2. the probability measure is unknown and the problem has to be solved on the data base. It means that the underlying probability measure $P_{F}$ has to be replaced by its statistical estimates, mostly by empirical distribution function;

3. the distribution function $F$ corresponds to real situation, however, the optimization problem (1.1) is (from the numerical point of view) very complicated. Consequently, the "underlying" distribution function has to be approximated by simpler one (usually the continuous function is replaced by discrete one). 
Evidently, in all these cases, two optimization problems (real and approximate) correspond to real applications. The relationship between these problems has been investigated in the stochastic programming literature many times, however mostly, in the case when the distribution $F$ has been replaced by empirical one (see, e.g., $1,2,7,8,11,19,21,22,24,25,26,30$ ). The aim of this paper is, first, to recall some of these results and furthermore to employ them to scenario generation. Moreover we try to employ these results to a simple financial problem.

The paper is organized as follows. First, we recall some definitions and auxiliary assertions (section 2). Section 3 is devoted to suitable results on the stability based on the Wasserstein metric corresponding to $\mathcal{L}_{1}$ norm and their applications to empirical estimates. Applications to scenario generation can be found in Section 4. Section 5 is devoted to an analysis of a simple financial model. The paper is ended by a short Conclusion (section 6).

\section{SOME DEFINITION AND AUXILIARY ASSERTION}

To recall suitable stability assertions we first recall some definitions. To this end, if $\mathcal{P}\left(\mathbb{R}^{s}\right)$ denotes the set of all (Borel) probability measures on $\mathbb{R}^{s}$, then we can define the system $\mathcal{M}_{1}^{1}\left(\mathbb{R}^{s}\right)$ by the relation:

$\mathcal{M}_{1}^{1}\left(\mathbb{R}^{s}\right):=\left\{\nu \in \mathcal{P}\left(\mathbb{R}^{s}\right): \int_{\mathbb{R}^{s}}\|z\|_{1} \mathrm{~d} \nu(z)<\infty\right\},\|\cdot\|_{1}:=\|\cdot\|_{1}^{s}$ denotes $\mathcal{L}_{1}$ norm in $\mathbb{R}^{s}$.

Evidently, if $P_{F}, P_{G} \in \mathcal{M}_{1}^{1}\left(\mathbb{R}^{s}\right)$ (and the corresponding problems (1.1) are well defined), then employing the triangular inequality we can obtain

$$
\left|\varphi\left(F, X_{F}\right)-\varphi\left(G, X_{G}\right)\right| \leq\left|\varphi\left(F, X_{F}\right)-\varphi\left(F, X_{G}\right)\right|+\left|\varphi\left(F, X_{G}\right)-\varphi\left(G, X_{G}\right)\right| .
$$

Defining quantil's vector $k_{F}(\alpha)$ and the set $\bar{X}(v), v \in \mathbb{R}^{s}$ by the relations

$$
\begin{aligned}
k_{F}(\alpha)= & \left(k_{F_{1}}\left(\alpha_{1}\right), \ldots, k_{F_{s}}\left(\alpha_{s}\right)\right), \alpha=\left(\alpha_{1}, \ldots, \alpha_{s}\right), \\
k_{F_{i}}\left(\alpha_{i}\right)= & \sup \left\{z_{i} \mid P_{F_{i}}\left\{\omega \mid z_{i} \leq \xi_{i}(\omega)\right\} \geq \alpha_{i}\right\}, \alpha_{i} \in(0,1), i=1, \ldots, s, \\
& \bar{X}(v)=\bigcap_{i=1}^{s}\left\{x \in X \mid \bar{g}_{i}(x) \leq v_{i}\right\}, v=\left(v_{1}, \ldots, v_{s}\right), v \in \mathbb{R}^{s},
\end{aligned}
$$

we can recall the following auxiliary assertions.

Lemma 2.1. (Kaňková [10]) Let $\bar{g}_{i}(x), i=1, \ldots, s$ be continuous functions defined on $\mathbb{R}^{n}, P_{F_{i}}, i=1, \ldots, s$ be absolutely continuous w. r. t. the Lebesgue measure on $\mathbb{R}^{1}$. Let, moreover, $X_{F}(\alpha)$ be defined by (1.2), then

$$
X_{F}(\alpha)=\bar{X}\left(k_{F}(\alpha)\right), \quad \alpha=\left(\alpha_{1}, \ldots, \alpha_{s}\right), \alpha_{i} \in(0,1), i=1, \ldots, s .
$$

$\left(F_{i}, i=1, \ldots, s\right.$ denote one-dimensional marginal distribution functions corresponding to $F$.) 
Lemma 2.2. Let $g(x, z), Y(z)$ be for every $x \in X$ a Lipschitz functions of $z \in \mathbb{R}^{s}$ with the Lipschitz constant $L_{g}$ not depending on $x \in X$. Let, moreover, $P_{F} \in \mathcal{M}_{1}^{1}\left(\mathbb{R}^{s}\right)$. If $X_{F}$ is defined by the relation (1.5), then

1.

$$
X_{F}=\left\{x \in X: \mathrm{E}_{F}(u-g(x, \xi))^{+} \leq \mathrm{E}_{F}(u-Y(\xi))^{+}, \quad u \in \mathbb{R}^{1}\right\}
$$

2.

$$
(u-g(x, z))^{+},(u-Y(z))^{+}, \quad u \in \mathbb{R}^{1}, x \in \mathbb{R}^{n}
$$

are Lipschitz functions of $z \in \mathbb{R}^{s}$ with the Lipschitz constant $L_{g}$ not depending on $u \in \mathbb{R}^{1}, x \in \mathbb{R}^{n}$.

Pro of. The first assertion follows from the relation (4.7) in [26], second assertion can be found in [14].

To recall assertions we introduce the system of assumptions.

A.0 $g_{0}(x, z)$ is for $x \in X$ a Lipschitz function of $z \in \mathbb{R}^{s}$ with the Lipschitz constant $L$ (corresponding to the $\mathcal{L}_{1}$ norm) not depending on $x$,

A.1 $g_{0}(x, z)$ is either a uniformly continuous function on $X \times \mathbb{R}^{s}$, or there exists $\varepsilon>0$ such that $g_{0}(x, z)$ is a function convex on $X^{\varepsilon}$ and bounded on $X^{\varepsilon} \times \mathbb{R}^{s}$, where $X^{\varepsilon}$ denotes the $\varepsilon-$ neighbourhood of the set $X$,

A.2 $-\left\{\xi^{i}\right\}_{i=1}^{\infty}$ is a sequence of independent random vectors corresponding to $F$,

- $F^{N}$ is an empirical distribution function determined by $\left\{\xi^{i}\right\}_{i=1}^{N}, N=1,2, \ldots$,

A.3 $P_{F_{i}}, i=1, \ldots, s$ are absolutely continuous w.r.t. the Lebesgue measure on $\mathbb{R}^{1}$ (we denote by $f_{i}, i=1, \ldots, s$ the probability densities corresponding to $F_{i}$ ),

A.4 there exist constants $\vartheta_{i}>0, \delta_{i}>0$ and $\delta_{i}-$ neighbourhood $U_{i}^{\delta_{i}}\left(k_{F_{i}}\left(\alpha_{i}\right)\right)$ of $k_{F_{i}}\left(\alpha_{i}\right)$ such that $f_{i}\left(z_{i}\right)>\vartheta_{i}$ for $z_{i} \in U_{i}^{\delta_{i}}\left(k_{F_{i}}\left(\alpha_{i}\right)\right), \alpha_{i} \in(0,1), i=1, \ldots, s$,

A.5 $\mathrm{E}_{F} g_{0}(x, \xi)$ is a Lipschitz function on $X$ with the Lipschitz constant $\bar{L}$.

\subsection{Stability}

First, we recall (for us) an important stability assertion.

Proposition 2.3. (Kaňková and Houda [1]) Let $P_{F}, P_{G} \in \mathcal{M}_{1}^{1}\left(\mathbb{R}^{s}\right)$. If Assumption A.0 is fulfilled, then

$$
\left|\mathrm{E}_{F} g_{0}(x, \xi)-\mathrm{E}_{G} g_{0}(x, \xi)\right| \leq L \sum_{i=1}^{s} \int_{-\infty}^{+\infty}\left|F_{i}\left(z_{i}\right)-G_{i}\left(z_{i}\right)\right| \mathrm{d} z_{i} \quad \text { for all } \quad x \in X
$$


If, moreover, $X$ is a compact set, A.1 is fulfilled, then also

$$
\left|\inf _{x \in X} \mathrm{E}_{F} g_{0}(x, \xi)-\inf _{x \in X} \mathrm{E}_{G} g_{0}(x, \xi)\right| \leq L \sum_{i=1}^{s} \int_{-\infty}^{+\infty}\left|F_{i}\left(z_{i}\right)-G_{i}\left(z_{i}\right)\right| \mathrm{d} z_{i} .
$$

If the assumptions of Lemma 2.1 are fulfilled, $X$ is a compact set, then $X_{F}, X_{G}$ defined by (1.2) are also compact sets. Assumptions under which it is possible to find out $\bar{C}>0$ such that

$$
\Delta\left[X_{F}(\alpha), X_{G}(\alpha)\right]=\Delta\left[\bar{X}\left(k_{F}(\alpha)\right), \bar{X}\left(k_{G}(\alpha)\right)\right] \leq \bar{C} \sum_{i=1}^{s}\left|k_{F_{i}}\left(\alpha_{i}\right)-k_{G_{i}}\left(\alpha_{i}\right)\right|
$$

are introduced in [10]. $\left(\Delta[\cdot, \cdot]=\Delta_{n}[\cdot, \cdot]\right.$ denotes the Hausdorff distance in the space of nonempty closed subsets of $\mathbb{R}^{n}$; for the definition of the Hausdorff distance see, e. g., 20 or 23.)

Lemma 2.4. Let $X$ be a nonempty compact set, $\alpha=\left(\alpha_{1}, \ldots, \alpha_{s}\right), \alpha_{i} \in(0,1), i=$ $1, \ldots, s$, Assumptions A.1, A.3 be fulfilled. If

1. $g_{0}(x, z)$ is a Lipschitz function on $X$ with the Lipschitz constant $\bar{L}$ not depending on $z \in Z_{F} \cup Z_{G}$,

2. $P_{F}, P_{G} \in \mathcal{M}_{1}^{1}\left(\mathbb{R}^{s}\right)$ and moreover $\bar{X}\left(k_{F}(\alpha)\right), \bar{X}\left(k_{G}(\alpha)\right)$ are nonempty sets,

3. there exists a constant $\bar{C}$ such that

$$
\Delta\left[\bar{X}\left(v^{1}\right), \bar{X}\left(v^{2}\right)\right] \leq \bar{C}\left\|v^{1}-v^{2}\right\|_{2} \quad \text { for every } \quad v^{1}, v^{2} \in Z_{F} \bigcup Z_{G},
$$

then

$$
\left|\inf _{x \in \bar{X}\left(k_{F}(\alpha)\right)} \mathrm{E}_{F} g_{0}(x, \xi)-\inf _{x \in \bar{X}\left(k_{G}(\alpha)\right)} \mathrm{E}_{F} g_{0}(x, \xi)\right| \leq \bar{L} \bar{C}\left\|k_{F}(\alpha)-k_{G}(\alpha)\right\|_{2} .
$$

$\left(\|\cdot\|_{2}:=\|\cdot\|_{2}^{s}\right.$ denotes the Euclidean norm in $\mathbb{R}^{s}$.)

P r o of. First, it follows from the Assumption 1 that $\mathrm{E}_{F} g_{0}(x, \xi)$ is a Lipschitz function on $X$ with the Lipschitz constant $\bar{L}$ (Assumption A.5). Consequently, it is easy to see that the assertion of Lemma 2.4 follows from Proposition 1 in [10].

Furthermore, evidently, if moreover Assumption A.4 is fulfilled and if

$$
\left|G_{i}\left(z_{i}\right)-F_{i}\left(z_{i}\right)\right| \leq \frac{1}{2} \vartheta_{i} \delta_{i} \quad \text { for } \quad z_{i} \in U_{i}^{\delta_{i}}\left(k_{F_{i}}\left(\alpha_{i}\right)\right), \quad i=1, \ldots, s,
$$

then

$$
G_{i}\left(k_{F_{i}}\left(\alpha_{i}\right)-\delta_{i}\right) \leq F_{i}\left(k_{F_{i}}\left(\alpha_{i}\right)\right)-\frac{1}{2} \vartheta_{i} \delta_{i}, \quad G_{i}\left(k_{F_{i}}\left(\alpha_{i}\right)+\delta_{i}\right) \geq F_{i}\left(k_{F_{i}}\left(\alpha_{i}\right)\right)+\frac{1}{2} \vartheta_{i} \delta_{i} .
$$

Consequently $k_{G_{i}}\left(\alpha_{i}\right) \in\left\langle k_{F_{i}}\left(\alpha_{i}\right)-\delta_{i}, k_{F_{i}}\left(\alpha_{i}\right)+\delta_{i}\right\rangle$ and

$$
\left|\inf _{x \in \bar{X}\left(k_{F}(\alpha)\right)} \mathrm{E}_{F} g_{0}(x, \xi)-\inf _{x \in \bar{X}\left(k_{G}(\alpha)\right)} \mathrm{E}_{F} g_{0}(x, \xi)\right| \leq \bar{L} \bar{C} \sum_{i=1}^{s} \delta_{i} .
$$

Summarizing now the relation (2.7), Proposition 2.3 and Lemma 2.4 we can obtain. 
Proposition 2.5. Let $X$ be nonempty compact set, $\alpha=\left(\alpha_{1}, \ldots, \alpha_{s}\right), \alpha_{i} \in(0,1), i=$ $1, \ldots, s, P_{F}, P_{G} \in \mathcal{M}_{1}^{1}\left(\mathbb{R}^{s}\right)$, Assumptions A.0, A.1, A.3 be fulfilled. If, moreover,

1. $g_{0}(x, z)$ is a Lipschitz function on $X$ with the Lipschitz constant $\bar{L}$ not depending on $z$,

2. $\bar{X}\left(k_{F}(\alpha)\right), \bar{X}\left(k_{G}(\alpha)\right)$ are nonempty sets,

3. there exists a constant $\bar{C}$ such that

$$
\Delta\left[\bar{X}\left(v^{1}\right), \bar{X}\left(v^{2}\right)\right] \leq \bar{C}\left\|v^{1}-v^{2}\right\|_{2} \quad \text { for every } \quad v^{1}, v^{2} \in Z_{F} \bigcup Z_{G},
$$

then

$$
\begin{aligned}
& \left|\inf _{x \in \bar{X}\left(k_{F}(\alpha)\right)} \mathrm{E}_{F} g_{0}(x, \xi)-\inf _{x \in \bar{X}\left(k_{G}(\alpha)\right)} \mathrm{E}_{G} g_{0}(x, \xi)\right| \leq \\
& L \sum_{i=1}^{s} \int_{-\infty}^{+\infty}\left|F_{i}\left(z_{i}\right)-G_{i}\left(z_{i}\right)\right| \mathrm{d} z_{i}+\bar{L} \bar{C}\left\|k_{F}(\alpha)-k_{G}(\alpha)\right\|_{2} .
\end{aligned}
$$

Analyzing further the case of constraints set $X_{F}$ introduced by (1.5) we first define for $\varepsilon \in \mathbb{R}^{1}$ the sets

$$
X_{F}^{\varepsilon}=\left\{x \in X: \mathrm{E}_{F}(u-g(x, \xi))^{+}-\mathrm{E}_{F}(u-Y(\xi))^{+} \leq \varepsilon, \quad u \in \mathbb{R}^{1}\right\}, \quad \varepsilon \in \mathbb{R}^{1} ;
$$

evidently $X_{F}^{0}=X_{F}$.

If the assumptions of Lemma 2.2 are fulfilled, $P_{F}, P_{G} \in \mathcal{M}_{1}^{1}\left(\mathbb{R}^{s}\right), u \in \mathbb{R}^{1}, x \in X$, then

$$
\begin{aligned}
\left|\mathrm{E}_{F}(u-g(x, \xi))^{+}-\mathrm{E}_{G}(u-g(x, \xi))^{+}\right| & \leq L_{g} \sum_{i=1}^{s} \int_{-\infty}^{+\infty}\left|F_{i}\left(z_{i}\right)-G_{i}\left(z_{i}\right)\right| \mathrm{d} z_{i}, \\
\left|\mathrm{E}_{F}(u-Y(\xi))^{+}-\mathrm{E}_{G}(u-Y(\xi))^{+}\right| & \leq L_{g} \sum_{i=1}^{s} \int_{-\infty}^{+\infty}\left|F_{i}\left(z_{i}\right)-G_{i}\left(z_{i}\right)\right| \mathrm{d} z_{i} .
\end{aligned}
$$

Consequently

$$
\begin{aligned}
& \begin{aligned}
\mid \mathrm{E}_{F}(u-g(x, \xi))^{+}+\mathrm{E}_{G}(u-g(x, \xi))^{+}-\mathrm{E}_{F}(u & -Y(\xi))^{+}-\mathrm{E}_{G}(u-Y(\xi))^{+} \mid \\
& \leq 2 L_{g} \sum_{i=1}^{s} \int_{-\infty}^{+\infty}\left|F_{i}\left(z_{i}\right)-G_{i}\left(z_{i}\right)\right| \mathrm{d} z_{i},
\end{aligned} \\
& x \in X_{F} \Rightarrow \mid \mathrm{E}_{G}(u-g(x, \xi))^{+}-\mathrm{E}_{G}\left(u-Y(\xi)^{+}\left|\leq 2 L_{g} \sum_{i=1}^{s} \int_{-\infty}^{+\infty}\right| F_{i}\left(z_{i}\right)-G_{i}\left(z_{i}\right) \mid \mathrm{d} z_{i},\right. \\
& x \in X_{G} \Rightarrow \mid \mathrm{E}_{F}(u-g(x, \xi))^{+}-\mathrm{E}_{F}\left(u-Y(\xi)^{+}\left|\leq 2 L_{g} \sum_{i=1}^{s} \int_{-\infty}^{+\infty}\right| F_{i}\left(z_{i}\right)-G_{i}\left(z_{i}\right) \mid \mathrm{d} z_{i}\right.
\end{aligned}
$$


or equivalently

$x \in X_{F} \Longrightarrow x \in X_{G}^{\varepsilon}, \quad x \in X_{G} \Longrightarrow x \in X_{F}^{\varepsilon} \quad$ with $\quad \varepsilon=2 L_{g} \sum_{i=1}^{s} \int_{-\infty}^{+\infty}\left|F_{i}\left(z_{i}\right)-G_{i}\left(z_{i}\right)\right| \mathrm{d} z_{i}$.

More generally

$$
X_{G}^{\delta-\varepsilon} \subset X_{F}^{\delta} \subset X_{G}^{\delta+\varepsilon} \text { for } \delta \in \mathbb{R}^{1}
$$

Lemma 2.6. Let $X$ be a nonempty compact set, $P_{F}, P_{G} \in \mathcal{M}_{1}^{1}\left(\mathbb{R}^{s}\right)$, Assumption A.1 be fulfilled. Let, moreover, $g(x, z)$ be for every $x \in X$ a Lipschitz function of $z \in Z_{F} \cup Z_{G}$ with the Lipschitz constant $L_{g}$ not depending on $x \in X$. If

1. $g_{0}(x, z)$ is a Lipschitz function on $X$ with the Lipschitz constant $\bar{L}$ not depending on $z$ (Assumption 5),

2. $X_{F}, X_{G}$ defined by (1.5) are nonempty compact sets,

3. there exists a constant $D>0$ such that

$$
\Delta\left[X_{F}^{\varepsilon^{\prime}}, X_{F}^{\varepsilon^{\prime \prime}}\right] \leq D \varepsilon \quad \text { for every } \quad \varepsilon^{\prime}, \varepsilon^{\prime \prime} \in\langle-3 \varepsilon, 3 \varepsilon\rangle
$$

$$
\text { with } \varepsilon=2 L_{g} \sum_{i=1}^{s} \int_{-\infty}^{+\infty}\left|F_{i}\left(z_{i}\right)-G_{i}\left(z_{i}\right)\right| \mathrm{d} z_{i}
$$

then

$$
\left|\inf _{x \in X_{F}} \mathrm{E}_{F} g_{0}(x, \xi)-\inf _{x \in X_{G}} \mathrm{E}_{F} g_{0}(x, \xi)\right| \leq 2 D \bar{L} L_{g} \sum_{i=1}^{s} \int_{-\infty}^{+\infty}\left|F_{i}\left(z_{i}\right)-G_{i}\left(z_{i}\right)\right| \mathrm{d} z_{i} .
$$

Pr o of. Since the Hausdorff distance is a metric in the space of compact subsets of $\mathbb{R}^{n}$, (see, e.g., 23]) we can obtain that

$$
\Delta\left[X_{F}, X_{G}\right] \leq \Delta\left[X_{F}, X_{F}^{-\varepsilon}\right]+\Delta\left[X_{F}^{-\varepsilon}, X_{G}\right]
$$

Further employing (2.17) and assumption 3 of Lemma 2.6 we can obtain

$$
\Delta\left[X_{F}, X_{G}\right] \leq 2 D \varepsilon
$$

The assertion of Lemma 2.6 now already follows from Proposition 1 in [10].

Employing the relation (2.7), Proposition 2.3 and Lemma 2.6 we can obtain. 
Proposition 2.7. Let $X$ be a compact set, $P_{F}, P_{G} \in \mathcal{M}_{1}^{1}\left(\mathbb{R}^{s}\right)$, Assumptions A.0, A.1 and A.3 be fulfilled. Let, moreover, $g(x, z)$ be for every $x \in X$ a Lipschitz function of $z \in Z_{F} \cup Z_{G}$ with the Lipschitz constant $L_{g}$ not depending on $x \in X$. If

1. $g_{0}(x, z)$ is a Lipschitz function on $X$ with the Lipschitz constant $L$ not depending on $z \in Z_{F} \cup Z_{G}$,

2. $X_{F}, X_{G}$ defined by (1.5) are nonempty compact sets,

3. there exists a constant $D>0$ such that

$$
\begin{gathered}
\Delta\left[X_{F}^{\varepsilon^{\prime}}, X_{F}^{\varepsilon^{\prime \prime}}\right] \leq D \varepsilon \quad \text { for every } \quad \varepsilon^{\prime}, \varepsilon^{\prime \prime} \in\langle-3 \varepsilon, 3 \varepsilon\rangle, \\
\text { with } \varepsilon=2 L_{g} \sum_{i=1}^{s} \int_{-\infty}^{+\infty}\left|F_{i}\left(z_{i}\right)-G_{i}\left(z_{i}\right)\right| \mathrm{d} z_{i},
\end{gathered}
$$

then there exists a constant $D^{\prime}>0$ such that

$$
\left|\inf _{x \in X_{F}} \mathrm{E}_{F} g_{0}(x, \xi)-\inf _{x \in X_{G}} \mathrm{E}_{G} g_{0}(x, \xi)\right| \leq D^{\prime} \sum_{i=1}^{s} \int_{-\infty}^{+\infty}\left|F_{i}\left(z_{i}\right)-G_{i}\left(z_{i}\right)\right| \mathrm{d} z_{i} .
$$

Remark 2.8. The constant $D^{\prime}$ in Proposition 2.7 can be estimated by the value $L+$ $3 D \bar{L} L_{g}$.

\subsection{Empirical estimates}

Since in applications very often $P_{F}$ has to be replaced by empirical $P_{F^{N}}$, the solution of (1.1) has to be (mostly) sought w.r.t. an "empirical problem":

Find

$$
\varphi\left(F^{N}, X_{F^{N}}\right)=\inf \left\{\mathrm{E}_{F^{N}} g_{0}(x, \xi) \mid x \in X_{F^{N}}\right\}
$$

where $F^{N}$ denotes an empirical distribution function determined by random sample $\left\{\xi^{i}\right\}_{i=1}^{N}$ (not necessarily independent) corresponding to $F$. If we denote by symbols $\mathcal{X}\left(F, X_{F}\right)$ and $\mathcal{X}\left(F^{N}, X_{F^{N}}\right)$ the optimal solution sets of (1.1) and (2.20), then under rather general assumptions, $\varphi\left(F^{N}, X_{F^{N}}\right), \mathcal{X}\left(F^{N}, X_{F^{N}}\right)$ are "good" statistical estimates of $\varphi\left(F, X_{F}\right), \mathcal{X}\left(F, X_{F}\right)$. The investigation of these estimates started in 1974 (see [30]) and it was followed by [7, 8] and many others. (Some of them were mentioned already in the Introduction, see also the subsection STABILITY.)

Results on the statistical estimates (in the stochastic programming literature) are often based on the large deviations (started by employing the inequality published in [4]), on the stability assertions corresponding to different distances in the spaces of the probability measures, see, e.g., 6, 8, 9, 19, 21, 22. In this paper we focus mostly on the case when the empirical estimates are based on the stability results and $\mathcal{L}_{1}$ norm and the results corresponding to quantils (see subsection STABILITY). Replacing $G$ by $F^{N}$ (in the stability results) we can investigate the relationship between $\varphi\left(F^{N}, X_{F^{N}}\right)$, 
$\mathcal{X}\left(F^{N}, X_{F^{N}}\right)$ and $\varphi\left(F, X_{F}\right), \mathcal{X}\left(F, X_{F}\right)$. We focus on the investigation of relationship between $\varphi\left(F, X_{F}\right)$ and $\varphi\left(F^{N}, X_{F^{N}}\right)$. To this end it is evidently suitable to investigate

$$
\int_{-\infty}^{\infty}\left|F_{i}\left(z_{i}\right)-F_{i}^{N}\left(z_{i}\right)\right| \mathrm{d} z_{i}, \quad\left|k_{F_{i}}\left(\alpha_{i}\right)-k_{F_{i}^{N}}\left(\alpha_{i}\right)\right|, \alpha_{i} \in(0,1), i=1, \ldots, s .
$$

Lemma 2.9. (Shorack and Welner [29]) Let $s=1, P_{F} \in \mathcal{M}_{1}^{1}\left(\mathbb{R}^{1}\right)$. Let, moreover, the assumption A.2 be fulfilled, then

$$
P\left\{\omega: \int_{-\infty}^{\infty}\left|F(z)-F^{N}(z)\right| \mathrm{d} z \underset{N \rightarrow \infty}{\longrightarrow} 0\right\}=1 .
$$

Evidently, the results of Lemma 2.9 hold (for one-dimensional random value) when the finite first moment exists. The case of convergence rate is more complicated. To investigate it we recall the following auxiliary assertion.

Lemma 2.10. (Houda and Kaňková [5]) Let $s=1, r>0, t>0$, Assumptions A.2, A.3 be fulfilled. Let, moreover, $\xi$ be a random variable such that $\mathrm{E}_{F}|\xi|^{r}<\infty$. If constants $\beta, \gamma>0$ fulfil the inequalities

$$
0<\beta+\gamma<1 / 2, \quad \gamma>1 / r, \quad \beta+(1-r) \gamma<0
$$

then

$$
P\left\{\omega: N^{\beta} \int_{-\infty}^{\infty}\left|F(z)-F^{N}(z)\right|>t\right\} \underset{N \rightarrow \infty}{\longrightarrow} 0
$$

Evidently, the convergence rate $\beta:=\beta(r)$ (introduced in Lemma 2.10) depends on the existence of finite absolutely moments. Unfortunately, we cannot obtain (by this approach) any results in the case when there exist only $\mathrm{E}_{F}|\xi|^{r}<\infty$ for $r<2$. Some weaker assertions (for this case) can be found in [5].

Lemma 2.11. (Kaňková 13 ) Let $s=1, \alpha \in(0,1)$. If Assumptions A.2, A.3 and A.4 are fulfilled, $0<t^{\prime}<\delta$, then

$$
P\left\{\omega:\left|k_{F}(\alpha)-k_{F^{N}}(\alpha)\right|>t^{\prime}\right\} \leq 2 \exp \left\{-2 N\left(\vartheta t^{\prime}\right)^{2}\right\}, \quad N \in \mathcal{N} .
$$

$\left(\mathcal{N}\right.$ denotes the set of natural numbers, $\delta=\delta_{1}, \vartheta=\vartheta_{1}$ defined by A.4.)

Employing Lemma 2.10, Lemma 2.11, Proposition 2.3, Proposition 2.5 and the properties of the exponentional function we obtain.

Proposition 2.12. Let $X$ be a compact set, Assumptions A.0, A.1, A.2, A.3 and A.4 be fulfilled, $\alpha=\left(\alpha_{1}, \ldots, \alpha_{s}\right), \alpha_{i} \in(0,1)$ and $t>0$. Let moreover $X_{F}$ be defined by the relation (1.2). If 
1. for every $v \in Z_{F}, \bar{X}(v)$ are nonempty sets and moreover there exists a constant $\bar{C}$ such that

$$
\Delta\left[\bar{X}\left(v^{1}\right), \bar{X}\left(v^{2}\right)\right] \leq \bar{C}\left\|v^{1}-v^{2}\right\|_{2}, \quad v^{1}, v^{2} \in Z_{F},
$$

2. $g_{0}(x, z)$ is for every $z \in Z_{F}$ a Lipschitz function of $x \in X$ with the Lipschitz constant $\bar{L}$ not depending on $z \in Z_{F}$ (Assumption A.5),

3. for all components $\xi_{i}, i=1, \ldots, s$ of the vector $\xi$ and $r>0$ there exist finite $\mathrm{E}_{F}\left|\xi_{i}\right|^{r}$. If constants $\beta, \gamma>0$ fulfil the inequalities

$$
0<\beta+\gamma<1 / 2, \quad \gamma>1 / r, \quad \beta+(1-r) \gamma<0
$$

then

$$
P\left\{\omega: N^{\beta}\left|\inf _{\bar{X}\left(k_{F}(\alpha)\right)} \mathrm{E}_{F} g_{0}(x, \xi)-\inf _{\bar{X}\left(k_{F^{N}}(\alpha)\right)} \mathrm{E}_{F^{N}} g_{0}(x, \xi)\right|>t\right\} \underset{N \rightarrow \infty}{\longrightarrow} 0 .
$$

Considering $X_{F}$ defined by (1.5) we can obtain.

Proposition 2.13. (Kaňková and Houda [14]) Let $X$ be a compact set, $P_{F} \in \mathcal{M}_{1}^{1}\left(\mathbb{R}^{s}\right)$, Assumptions A.0, A.1, A.2 and A.3 be fulfilled, $X_{F}$ be defined by the relation (1.5). Let, moreover, $g(x, z)$ be for every $x \in X$ a Lipschitz function of $z \in Z_{F}$ with the Lipschitz constant not depending on $x \in X$. If

1. $g_{0}(x, z)$ is a Lipschitz function on $X$ with the Lipschitz constant $L$ not depending on $z \in Z_{F}$,

2. $X_{F}$ defined by (1.5) is a nonempty compact set,

3. there exists $\varepsilon_{0}>0$ such that $X_{F}^{\varepsilon}$ are nonempty compact sets for every $\varepsilon \in\left\langle-\varepsilon_{0}, \varepsilon_{0}\right\rangle$ and, moreover, there exists a constant $\hat{C}>0$ such that

$$
\Delta\left[x_{F}^{\varepsilon}, X_{F}^{\varepsilon^{\prime}}\right] \leq \hat{C}\left|\varepsilon-\varepsilon^{\prime}\right| \quad \text { for } \quad \varepsilon, \varepsilon^{\prime} \in\left\langle-\varepsilon_{0}, \varepsilon_{0}\right\rangle,
$$

4. for all components $\xi_{i}, i=1, \ldots, s$ of the vector $\xi$ and $r>0$ there exist finite $\mathrm{E}_{F}\left|\xi_{i}\right|^{r}$. If constants $\beta, \gamma>0$ fulfil the inequalities

$$
0<\beta+\gamma<1 / 2, \quad \gamma>1 / r, \quad \beta+(1-r) \gamma<0,
$$

then

$$
P\left\{\omega: N^{\beta}\left|\inf _{X_{F}} \mathrm{E}_{F} g_{0}(x, \xi)-\inf _{X_{F^{N}}} \mathrm{E}_{F^{N}} g_{0}(x, \xi)\right|>t\right\} \underset{N \rightarrow \infty}{\longrightarrow} 0 .
$$




\section{SCENARIO GENERATION}

Evidently, it can be very complicated (from the numerical point of view) to solve the stochastic programming problems; especially, when the "underlying" probability measure belongs to a continuous type. Consequently the "underlying" continuous probability measure is often replaced by discrete one with finite number of atoms (scenaria). We employ the results on the stability (subsection 2.1) to suggest one of possibilities to scenario generation. First, we consider the case of static (one-stage) stochastic programming problems, further we try to generalize this approach to special case of multistage stochastic programming problem.

\subsection{One--stage case}

First, we shall deal with the case of static stochastic programs. In particular, the aim of this subsection is to deal with the above mentioned discrete approximation in the case when the approximation error can be estimated by the sum of one-dimensional Wasserstein distances. To this end we employ the stability results. Namely considering Problem (1.1) with $X_{F}=X$ or with $X_{F}$ fulfilling the definition (1.5) and assuming that $X$ is a compact set, A.0, A.1 are fulfilled and $P_{F}, P_{G} \in \mathcal{M}_{1}^{1}\left(\mathbb{R}^{s}\right)$ we can see that

$$
\left|\varphi\left(F, X_{F}\right)-\varphi\left(G, X_{G}\right)\right|
$$

can be bounded by the value

$$
L^{\prime} \sum_{i=1}^{s} \int_{-\infty}^{+\infty}\left|F_{i}\left(z_{i}\right)-G_{i}\left(z_{i}\right)\right| \mathrm{d} z_{i}, \quad L^{\prime} \quad \text { "suitable" constant. }
$$

Consequently, if we suppose that $F$ is "underlying original" distribution function and $G$ approximate one, then evidently we can construct $G$ with given approximation error. To this end we employ the approach of [15]. If A.3 is fulfilled, $P_{F_{i}} \in \mathcal{M}_{1}^{1}\left(\mathbb{R}^{1}\right), i=1, \ldots, s$, then for given $M_{i}>0, i=1, \ldots, s$ there exist natural numbers $m_{i}, i=1, \ldots, s$, points $z_{i, j}, \in \overline{\mathbb{R}}^{1}, j=0,1, \ldots, m_{i}$ and one-dimensional discrete distribution functions $G_{i}$ such that

$$
-\infty=z_{i, 0}<z_{i, 1}<z_{i, 2}<\ldots<z_{i, m_{i-1}}<z_{i, m_{i}}=\infty
$$

and, simultaneously,

$$
(L / s) \int_{-\infty}^{\infty}\left|F_{i}\left(z_{i}\right)-G_{i}\left(z_{i}\right)\right| \mathrm{d} z_{i} \leq M_{i}, i=1, \ldots, s .
$$

$\left(\overline{\mathbb{R}}^{1}\right.$ denotes the extended real line.)

Furthermore, it follows from the last relations that there exists $s$-dimensional distribution function $G$ with marginals $G_{i}, i=1, \ldots, s$ such that

$$
L \sum_{i=1}^{s} \int_{-\infty}^{+\infty}\left|F_{i}\left(z_{i}\right)-G_{i}\left(z_{i}\right)\right| \mathrm{d} z_{i} \leq \sum_{i-1}^{s} M_{i}
$$


Employing the relation (3.24), Proposition 2.3 and Proposition 2.7, we can see that the following Proposition is valid.

Proposition 3.1. Let Assumption A.0, A.1, A.3 be fulfilled, $P_{F_{i}} \in \mathcal{M}_{1}^{1}\left(\mathbb{R}^{1}\right)$. Let, moreover, $M, \bar{M}>0, X$ be a compact set. If

1. $X_{F}=X$, then there exists a discrete distribution function $G$ with discrete marginals $G_{i}, i=1, \ldots, s$ and finite number of atoms such that

$$
|\varphi(F, X)-\varphi(G, X)| \leq M
$$

2. $-X_{F}$ fulfils the relation (1.5) and the assumption 2 of Lemma 2.12 holds,

- $g(x, z)$ is for every $x \in X$ a Lipschitz function of $z \in Z_{F}$ with the Lipschitz constant $L_{g}$ not depending on $x \in X$,

- there exists $\varepsilon_{0}, \hat{D}>0$ such that

$$
\Delta\left[X_{F}^{\varepsilon}, X_{F}^{\varepsilon^{\prime}}\right] \leq \hat{D}\left|\varepsilon-\varepsilon^{\prime}\right| \quad \text { for } \quad \varepsilon, \varepsilon^{\prime} \in\left\langle-3 \varepsilon_{0}, 3 \varepsilon_{0}\right\rangle,
$$

then there exists a discrete distribution function $\bar{G}$ with finite number of atoms such that

$$
\left|\varphi\left(F, X_{F}\right)-\varphi\left(\bar{G}, X_{\bar{G}}\right)\right| \leq \bar{M}
$$

\section{Remark 3.2.}

- According to Proposition 2.7 it is suitable the following relation

$$
\varepsilon_{0} \geq 2 L_{g} \sum_{i=1}^{s} \int_{-\infty}^{+\infty}\left|F_{i}\left(z_{i}\right)-\bar{G}_{i}\left(z_{i}\right)\right| \mathrm{d} z_{i}
$$

be fulfilled.

- In the case when $X_{F}$ fulfils the relation (1.2), then it is necessary to consider the case 1 , to assume A.4, A.5, $L \bar{C}\left\|k_{F}(\alpha)-k_{G}(\alpha)\right\|_{2}$ be sufficiently small, employ the relation (2.7) and Proposition 2.5.

\subsection{Multistage case}

Till now we have considered problems with respect to one time point. However economic activities are mostly developing in time and moreover it is reasonable to determine a decision (in given time point) as a function of a random sequence realization and decision to this time. Multistage stochastic programming problems pose to such situation. In this paper we consider only special case of the multistage programs. To this end we recall $T+1-$ stage stochastic programs by the following way:

Find

$$
\varphi_{\mathcal{F}}(T)=\inf \left\{\mathrm{E}_{F^{\xi^{0}}} g_{\mathcal{F}}^{0}\left(x^{0}, \xi^{0}\right) \mid x^{0} \in \mathcal{K}^{0}\right\},
$$


where the function $g_{\mathcal{F}}^{0}\left(x^{0}, z^{0}\right)$ is defined recursively

$$
\begin{aligned}
& g_{\mathcal{F}}^{k}\left(\bar{x}^{k}, \bar{z}^{k}\right) \quad=\quad \inf \left\{\mathrm{E}_{F^{\xi^{k+1} \mid \bar{\xi}^{k}=\bar{z}^{k}}} g_{\mathcal{F}}^{k+1}\left(\bar{x}^{k+1}, \bar{\xi}^{k+1}\right) \mid x^{k+1} \in \mathcal{K}_{\mathcal{F}}^{k+1}\left(\bar{x}^{k}, \bar{z}^{k}\right)\right\}, \\
& k=0,1, \ldots, T-1, \\
& g_{\mathcal{F}}^{T}\left(\bar{x}^{T}, \bar{z}^{T}\right):=\quad g_{0}^{T}\left(\bar{x}^{T}, \bar{z}^{T}\right), \quad \mathcal{K}_{0}:=X^{0} .
\end{aligned}
$$

$\xi^{j}:=\xi^{j}(\omega), j=0,1, \ldots, T$ denotes an $s$-dimensional random vector defined on a probability space $(\Omega, \mathcal{S}, P) ; F^{\xi^{j}}\left(z^{j}\right), z^{j} \in \mathbb{R}^{s}, j=0,1 \ldots, T$ the distribution function of $\xi^{j}$ and $F^{\xi^{k} \mid \bar{\xi}^{k-1}}\left(z^{k} \mid \bar{z}^{k-1}\right), z^{k} \in \mathbb{R}^{s}, \bar{z}^{k-1} \in \mathbb{R}^{(k-1) s}, k=1, \ldots, T$ the conditional distribution function $\left(\xi^{k}\right.$ conditioned by $\left.\bar{\xi}^{k-1}\right) ; P_{F^{j}}, P_{F^{\xi^{k+1}} \mid \bar{\xi}^{k}}, j=0,1, \ldots, T, k=$ $0,1, \ldots, T-1$ the corresponding probability measures; $Z^{F^{j}}:=Z_{F^{j}} \subset \mathbb{R}^{s}, j=0,1, \ldots, T$ the support of the probability measure $P_{F^{\xi}}$. Furthermore, the symbol $g_{0}^{T}:=g_{0}^{T}\left(\bar{x}^{T}, \bar{z}^{T}\right)$ denotes a continuous function defined on $\mathbb{R}^{n(T+1)} \times \mathbb{R}^{s(T+1)} ; X^{k} \subset \mathbb{R}^{n}, k=0,1, \ldots, T$ is a nonempty compact set; the symbol $\mathcal{K}_{\mathcal{F}}^{k+1}\left(\bar{x}^{k}, \bar{z}^{k}\right):=\mathcal{K}_{F^{k+1} \bar{\xi}^{k}}^{k+1}\left(\bar{x}^{k}, \bar{z}^{k}\right), k=0,1, \ldots$ $\ldots, T-1$ denotes a measurable multifunction defined on $\mathbb{R}^{n(k+1)} \times \mathbb{R}^{s(k+1)}$ with "values" subsets of $\mathbb{R}^{n} \cdot \bar{\xi}^{k}\left(:=\bar{\xi}^{k}(\omega)\right)=\left[\xi^{0}, \ldots, \xi^{k}\right] ; \bar{z}^{k}=\left[z^{0}, \ldots, z^{k}\right], z^{j} \in \mathbb{R}^{s} ; \bar{x}^{k}=$ $\left[x^{0}, \ldots, x^{k}\right], x^{j} \in \mathbb{R}^{n} ; \bar{X}^{k}=X^{0} \times X^{1} \ldots \times X^{k} ; \bar{Z}^{k}:=\bar{Z}_{\mathcal{F}}^{k}=Z_{F^{\xi^{0}}} \times Z_{F^{\xi^{1}}} \ldots \times Z_{F^{k}}$, $j=0,1, \ldots, k, k=0,1, \ldots, M$. Symbols $\mathrm{E}_{F^{\xi^{0}}}, \mathrm{E}_{F^{\xi^{k+1} \mid \bar{\xi}^{k}=\bar{z}^{k}}}, k=0,1, \ldots, T-1$ denote the operators of mathematical expectation corresponding to $F^{\xi^{0}}, F^{\xi^{k+1}} \mid \bar{\xi}^{k}=\bar{z}^{k}, k=$ $0, \ldots, T-1$.

In the multistage case, we restrict to the case when the following assumption is fulfilled:

C.1 Random sequence $\left\{\xi^{k}\right\}_{k=-\infty}^{\infty}$ follows (generally) nonlinear autoregressive sequence

$$
\xi^{k}=H\left(\xi^{k-1}, \nu^{k}\right)
$$

where $\xi^{0}, \nu^{k}, k=1,2, \ldots$ are stochastically independent $s$-dimensional random vectors defined on $(\Omega, \mathcal{S}, P)$ and, moreover, $\nu^{k}, k=1, \ldots$ identically distributed. $H=\left(H_{1}, \ldots, H_{s}\right)$ is a Lipschitz vector function defined on $\mathbb{R}^{s}$. We denote the distribution function corresponding to $\nu^{1}=\left(\nu_{1}^{1}, \ldots, \nu_{s}^{1}\right)$ by the symbol $F^{\nu}$ and suppose the realization $\xi^{0}$ to be known.

Evidently, the multistage stochastic programming problem (3.27), (3.28) depends essentially on a system of (generally) conditional distribution functions

$$
\mathcal{F}=\left\{F^{\xi^{0}}\left(z^{0}\right), F^{\xi^{k} \mid \bar{\xi}^{k-1}}\left(z^{k} \mid \bar{z}^{k-1}\right), k=1, \ldots, T\right\} .
$$

Consequently, if we replace $\mathcal{F}$ by another system $\mathcal{G}$

$$
\mathcal{G}=\left\{G^{\xi^{0}}\left(z^{0}\right), G^{\xi^{k} \mid \bar{\xi}^{k-1}}\left(z^{k} \mid \bar{z}^{k-1}\right), k=1, \ldots, T\right\}
$$

we obtain another multistage stochastic programming problem with the optimal value denoted $\varphi_{\mathcal{G}}(T)$. 
Under Assumption C.1 the system $\mathcal{F}$ is determined by $F^{\xi^{0}}$ and $F^{\nu}$. Consequently, if we replace these two probability distribution functions by another $G^{\xi^{0}}$ and $G^{\nu}$, we obtain another system $\mathcal{G}$.

Considering, furthermore, the constraint sets $\mathcal{K}_{\mathcal{F}}^{k+1}\left(\bar{x}^{k}, \bar{z}^{k}\right), k=0, \ldots, T-1$ not depending on the probability measure, then the assumptions under which

$$
\left|\varphi_{\mathcal{F}}(M)-\varphi_{\mathcal{G}}(M)\right| \leq \sum_{i=1}^{s} C_{W}^{i} \int_{R^{1}}\left|F_{i}^{\nu}\left(z_{i}\right)-G_{i}^{\nu}\left(z_{i}\right)\right| \mathrm{d} z_{i}, \quad C_{W}^{i}>0, i=1, \ldots, s
$$

can be found in 12 . Consequently, if we define discrete distributions $G^{\xi_{0}}, G^{\nu}$ determined by the approach of Proposition 3.1 (the case 1), then we have an approximating system $\mathcal{G}$ given by discrete mostly conditional distributional functions. Furthermore, it follows from results of the above mentioned work that this approach can be generalized to the case when constraints sets are given by the individual probability constraints (for more details see, e.g., [12]).

Remark 3.3. In this subsection we have denoted only to deterministic scenario generation. However random scenario we can obtain by random sample. The corresponding property of such approximation are given in the subsection "Empirical Estimation".

\section{SIMPLE FINANCIAL PROBLEM}

We analyze very simple financial problem from the point of view of the stochastic programming theory. The aim of this example is not only to demonstrate a utilizing the former theory but also to present a life situation when this theory can not be completely employed. To this end we consider a situation about a mortgage and its instalments. Let us start with a common situation. People try to gain (in the last decades) own residence (a flat or little house). Since young people do not posses necessary financial resources, the bank sector offers them a mortgage. Of course banks can employ excellent experts to minimize their risk and maximize profit in dependence of debtor's position. The aim of our approach is to analyze the situation from the second side. In particular, our aim is to investigate the possibilities of the debtors not only in dependence on their present-day situation, but also on their future private and subjective decisions and on possible "unpleasant" events. In details the aim is to suggest a method for a recognition of a "safe" loan and simultaneously to offer tactics to state a plausible environment for future time. Of course we suppose that our analysis is first contribution to this situation. The stochastic programming theory will be employed to it. Let us start with simple standard situation. A young married couple wants to gain own flat. They already obtained an offer from banks determined by their present-day situation. However they have subjective plans. According to this fact we try to analyze their possibilities. To this end let us assume that (in the start time) their monthly income is

$$
Z_{0}=U_{0}+V_{0}, \quad \text { where } U_{0} \text { is an income of husband and } V_{0} \text { is an income of wife. }
$$

Evidently, this income can be divided into three parts $Z_{0}^{1}, Z_{0}^{2}, Z_{0}^{3}$, where $Z_{0}^{1}$ denotes means for a basic consumption, $Z_{0}^{2}$ denotes means that can be employed for a repayment 
of installments and $Z_{0}^{3}$ can be considered as an allocation to saving. Consequently

$$
Z_{0}=Z_{0}^{1}+Z_{0}^{2}+Z_{0}^{3}, \quad Z_{0}^{1}, Z_{0}^{2}>0, Z_{0}^{3} \geq 0 .
$$

Given the annuity repayments, which is the most standard way of repaying the loan; if we denote by a symbol $M$ the value of the loan, by $m$ number of identical installments and by $\zeta$ the loan interest rate, then the identical installments $b(M):=b(\zeta)$ in time points $t=1,2, \ldots, m$ (see, e.g., [17] or [28]) are given by

$$
\begin{aligned}
& b(M):=b(\zeta)=\frac{M \zeta}{1-v^{m}}, \quad \zeta \neq 0, \quad v=v(\zeta)=(1+\zeta)^{-1}, \\
& \frac{1}{m}, \quad \zeta=0 \text {. }
\end{aligned}
$$

It follows from the relations (4.31), (4.32) that (in the case when $\zeta \neq 0$ ) it is desirable (in "static" approach) the following inequality

$$
\frac{M \zeta(1+\zeta)^{m}}{(1+\zeta)^{m}-1} \leq Z_{0}^{2}
$$

to be fulfilled. Of course, this condition (in the extreme case) can be replaced by the inequality

$$
\frac{M \zeta(1+\zeta)^{m}}{(1+\zeta)^{m}-1} \leq Z_{0}^{2}+Z_{0}^{3}
$$

If it is possible to assume that the relations (4.31), (4.33) will be fulfilled also in future, then the young people can take the loan equal to the maximal value $M$ for which the inequality (4.33) (respectively (4.34)) is fulfilled. However mostly it is necessary to assume that the financial situation of young married couple can change. For example: it is reasonable to assume that in some time period, say $\left(m_{1}, m_{2}\right), 0<m_{1}<m_{2} \leq m$ the married couple plan to have a baby. According to this fact and to the social politics of a state the young people can assume the less income in this time, approximately equal to

$$
Z_{1}=U_{0}+V_{1}=Z_{0}^{1}+Z_{1}^{2}+Z_{1}^{3}, \quad Z_{1}^{2}, Z_{1}^{3} \geq 0, Z_{1}^{2} \leq Z_{0}^{2},
$$

where $V_{1}$ is the supposed income of wife in the time interval $\left\langle m_{1}, m_{2}\right\rangle ; Z_{1}^{2}$ denotes the means, that can be employed for a repayment of installments (of course $Z_{1}^{2} \leq Z_{0}^{2}$ ) and $Z_{1}^{3}$ saved amount in every year of this time interval (of course mostly $0 \leq Z_{1}^{3} \leq Z_{0}^{3}$ ). Evidently without financial reserve the inequalities

$$
Z_{0}^{1}+Z_{0}^{2} \leq U_{0}+V_{1}
$$

need to be fulfilled. Consequently, if

$$
U_{0}+V_{1}<Z_{0}^{1}+Z_{0}^{2},
$$

then a very serious trouble could arise. However, if the young couple saved every time point $t \in\left\{0, \ldots, m_{1}-1\right\}$ the amount $Z_{0}^{3}$ and if the inequality

$$
\frac{\left(m_{2}-m_{1}\right) M\left[\zeta(1+\zeta)^{m}\right]}{(1+\zeta)^{m}-1} \leq\left(m_{2}-m_{1}\right)\left[Z_{0}^{2}-Z_{1}^{2}\right]+\left(m_{1}-1\right) Z_{0}^{3}
$$


is fulfilled, then they endure the time period $\left\langle m_{1}, m_{2}\right\rangle$ without financial troubles.

To construct the relation (4.35), it has been assumed that the amount $Z_{0}^{3}$ is deterministic, the same in every time point $t \in\left\{0, \ldots, m_{1}-1\right\}$ and that this amount can not be changed. However this situation can be a little different. To explain a new approach we suppose $m_{1}=2, \quad m_{2}-m_{1}=2$; it means $m_{1}=2, m_{2}=4$ and $m$ is determined by the relation (4.32) (consequently dependent on $M$ ). Furthermore we denote $Z_{t}^{2}, t=0, \ldots m$ the means that can be employed for a repayment of installments, $Z_{t}^{3}$ an allocation for a saving $\left(0 \leq Z_{t}^{2} \leq Z_{0}^{2}, t=m_{1}, \ldots, m_{2}, Z_{t}^{3} \geq 0, t=0, \ldots, m\right)$.

We consider two special cases.

D 1. The deterministic value $Z_{0}^{3}$ (in the relation $(4.31)$ ) can be replaced by random values $Z_{t}^{3} ; Z_{t}^{3}, t \in\{0,1, \ldots, m\}$ with probability one non negative. Consequently the deterministic income $Z_{0}=Z_{0}^{1}+Z_{0}^{2}+Z_{0}^{3}$ is replaced by random $Z_{t}=Z_{0}^{1}+Z_{t}^{2}+$ $Z_{t}^{3}$ in all points $t=0,1, \ldots, m$. We assume that young people can these random amount (in time point $t=0,1$ ) invest (for example) into two assets to obtain:

in the original year the value

under the assumptions

$$
\begin{aligned}
& \xi_{0,1} x_{0,1}+\xi_{0,2} x_{0,2} \\
& x_{0,1}+x_{0,2} \leq Z_{0}^{3}, \quad x_{0,1}, x_{0,2} \geq 0
\end{aligned}
$$

in the second year the value under the assumptions

$$
\begin{aligned}
& \xi_{1,1} x_{1,1}+\xi_{1,2} x_{1,2} \\
& x_{1,1}+x_{1,2} \leq Z_{1}^{3}, \quad x_{1,1}, x_{1,2} \geq 0
\end{aligned}
$$

(under the assumptions that the profit obtained in the time $t=0$ can not influence the invested amount in the time $t=1$ ). Evidently, it is desirable (for young people under the assumption $Z_{2}^{2}=Z_{3}^{2}=Z_{4}^{2}$ ) the fulfilling of the relation

$$
\frac{\left(m_{2}-m_{1}\right) M\left[\zeta(1+\zeta)^{m}\right]}{(1+\zeta)^{m}-1} \leq 3\left[Z_{0}^{2}-Z_{2}^{2}\right]+\sum_{t=0}^{1}\left[\xi_{t, 1} x_{t, 1}+\xi_{t, 2} x_{t, 2}\right],
$$

and of course the maximization of a possible profit.

$Z_{0}^{3}, Z_{1}^{3}, \xi_{0,1}, \xi_{0,2}, \xi_{1,2}, \xi_{1,2}$ are generally supposed to be random variables with "positive support". Consequently, it is necessary to "specify" the sense of relations in D.1. In details, it is necessary to "specify" when the operator of mathematical expectation, probability constraints, risk constraints or stochastic dominance constraints are employed in the optimization problems. We set (for simplicity) to this case a very simple stochastic optimization problem.

$$
\text { Find } \quad \max M
$$

under the system of constraints

$$
\frac{M \zeta(1+\zeta)^{m}}{(1+\zeta)^{m}-1} \leq Z_{0}^{2}
$$




$$
\begin{aligned}
& P_{F}\left\{x_{t, 1}+x_{t, 2} \leq Z_{t}^{3}\right\} \geq 1-\varepsilon_{t}, \quad \varepsilon_{t} \in(0,1), x_{t, 1}, x_{t, 2} \geq 0, \quad t=0,1 \\
& P_{F}\left\{\frac{3 M\left[\zeta(1+\zeta)^{m}\right.}{(1+\zeta)^{m}-1} \leq \sum_{t=2}^{4}\left[Z_{0}^{2}-Z_{t}^{2}\right]+\sum_{t=0}^{1}\left[\xi_{t, 1} x_{t, 1}+\xi_{t, 2} x_{t, 2}\right]\right\} \geq 1-\varepsilon_{0} \\
& \\
& \varepsilon_{0} \in(0,1) .
\end{aligned}
$$

Evidently, in this case it is reasonable to add to an objective function (4.37) the second one

$$
\mathrm{E}_{F} \sum_{t=m_{1}}^{m}\left[\xi_{t, 1} x_{t, 1}+\xi_{t, 2} x_{t, 2}\right]
$$

with the corresponding constraints

$$
\begin{aligned}
P_{F}\left\{x_{t, 1}+x_{t, 2} \leq \max \left(0, Z_{t}^{3}\right)\right\} \geq & 1-\varepsilon_{t}, \varepsilon_{t} \in(0,1), x_{t, 1}, x_{t, 2} \geq 0, \\
& t=2,3,4, \\
P_{F}\left\{x_{t, 1}+x_{t, 2} \leq Z_{t}^{3}\right\} \quad \geq & 1-\varepsilon_{t}, \varepsilon_{t} \in(0,1), x_{t, 1}, x_{t, 2} \geq 0, \\
& t=5, \ldots, m .
\end{aligned}
$$

$\xi_{t, 1}, \xi_{t, 2}, t=m_{1}, \ldots, m$ random value. Consequently, we have constructed two objective stochastic programming problem with objective (4.37) and (4.41) and constraints (4.38), (4.39), (4.40) and (4.42). Analyzing this model we can see

- constraints (4.38) are linear deterministic,

- constraints (4.39) and (4.42) (according to Lemma 2.1) can be expressed in equivalent form of linear inequalities,

- (4.37) and (4.41) are linear objective functions. Employing a convex combination we obtain only one linear objective.

Evidently, mentioned objective and constraints are suitable for discrete approximation. However, it remains to deal with constraints set (4.40). This condition can be rewritten in the equivalent form

$$
P_{F}\left\{\sum_{t=2}^{4}\left[Z_{t}^{2}-Z_{0}^{2}\right] \leq \sum_{\substack{t=0 \\ \varepsilon_{0} \in(0,1)}}^{1}\left[\xi_{t, 1} x_{t, 1}+\xi_{t, 2} x_{t, 2}\right]-\frac{3 M\left[\zeta(1+\zeta)^{m}\right.}{(1+\zeta)^{m}-1}\right\} \geq 1-\varepsilon_{0}
$$

There the function in the probability is not simultaneously, in decision parameter and random factor, convex. Consequently, the corresponding constraint is not generally 
convex. It is question if it is not reasonable to employ Markowitz approach (for more details see, e.g. [3]) and to replace (4.43). by

$$
\mathrm{E}_{F} \sum_{t=0}^{1}\left[\xi_{t, 1} x_{i, 1}+\xi_{t, 2} x_{t, 2}\right]-K V\left[\sum_{i=0}^{1}\left[\xi_{t, 1} x_{t, 1}+\xi_{t, 2} x_{t, 2}\right] \geq \sum_{t=2}^{4}\left[Z_{t}^{2}-Z_{0}^{2}\right]\right.
$$

where $V$ denotes a symbol for the Variation, $K>0$ i s a suitable constant. How to take the constant $K$ is however evidently beyond the scope of this paper.

D.2 $Z_{t}^{3}(1), t=0,1, \ldots, m$ have a deterministic character. Let us assume that these amounts can be invested into two assets (portfolio) with random returns $\bar{\xi}_{t, 1}, \bar{\xi}_{t, 2}$. Mathematically saying, it is desirable to determine $x_{0,1}, x_{0,2}, x_{1,1}, x_{1,2}$ fulfilling the relations

$$
\begin{array}{ll} 
& x_{t, 1}+x_{t, 2} \leq Z_{t}^{3}, \quad x_{t, 1}, x_{t, 2} \geq 0, \\
& \hat{g}_{t}=\bar{\xi}_{t, 1} x_{t, 1}+\bar{\xi}_{t, 2} x_{t, 2}, \\
& t=0,1, \ldots, m .
\end{array}
$$

Evidently, it is possible also to define random values $Y_{t}$, by the following relation

$$
Y_{t}=\frac{1}{2} \bar{\xi}_{t, 1}+\frac{1}{2} \bar{\xi}_{t, 2}, \quad t=0,1, \ldots, m .
$$

$\hat{g}_{t}, Y_{t}$ are random values "depending" on $Z_{t}^{3}$.

Employing the theory of the stochastic dominance [26] it is "reasonable" to determine $x_{t, 1}, x_{t, 2}$ such that

$$
F_{\hat{g}_{t}} \succeq_{1} F_{Y_{t}}, \quad \text { or } \quad F_{\hat{g}_{t}} \succeq_{2} F_{Y_{t}}, \quad t=0,1, \ldots, m .
$$

Evidently, in this case we can construct the following optimization problem.

$$
\text { Find } \quad \max M
$$

under the system of constraints

$$
\begin{gathered}
\frac{M \zeta(1+\zeta)^{m}}{(1+\zeta)^{m}-1} \leq Z_{0}^{2}, \\
F_{\hat{g}_{t}} \succeq_{2} F_{Y_{t}}, \quad t=0,1 \\
\left.P_{F}\left\{\frac{3 M \zeta(1+\zeta)^{m}}{(1+\zeta)^{m}-1}\right\} \leq \sum_{t=2}^{4}\left[Z_{0}^{2}-Z_{t}^{2}\right]+\sum_{t=0}^{1}\left[\bar{\xi}_{t, 1} x_{t, 1}+\bar{\xi}_{t, 2} x_{t, 2}\right]\right\} \geq 1-\varepsilon_{0}, \\
\varepsilon_{0} \in(0,1) .
\end{gathered}
$$

It is reasonable to add to an objective function (4.47) the second one 


$$
\mathrm{E}_{F} \sum_{t=5}^{m}\left[\bar{\xi}_{t, 1} x_{t, 1}+\bar{\xi}_{t, 2} x_{t, 2}\right]
$$

with the corresponding constraints

$$
F_{\hat{g}_{t}} \succeq_{2} F_{Y_{t}}, \quad t=2, \ldots, m .
$$

Analyzing the approach D.2 we can see that in the constraints (4.49) and (4.52) the "underlying" distribution function can be replaced by discrete one. However, the constraint (4.50)) appears. This constraint is not convex, and evidently it has to be replaced by another. However for philosophy of the problem this condition is very important.

Remark 4.1. We have tried (in the last example) to analyze a situation of young married couple and their problem with mortgage. We have included in the model their private plans (to have baby), however we have neglected many troubles and situations that can happen (e.g. illness, a loss of employment). But we also omitted a possibility to gain "better" career or only increasing salary. Every of these possibilities are waiting to be included.

\section{CONCLUSION}

The aim of the paper is to summarize a possibility to employ the results on the stability (based on the Wassersten metric and $\mathcal{L}_{1}$ norm) to some other parts of stochastic programming. In particular, stochastic programming problems with "deterministic" constraints, individual probability constraints and stochastic dominance constraints are considered. To introduce the survey of possible applications, first, the corresponding stability results are recalled. Further, the stability results are employed to empirical estimates in the stochastic programming and scenario generation. The paper is finished with a simple financial problem.

Evidently, by this approach a stochastic dependence between components of the random element is neglected. However, all formulas are simple and, moreover, they are acceptable from the numerical point of views. The idea to reduce $s$-dimensional case to one-dimensional case is credited to G. Pflug [19] (see also [27]).

A very simple example is presented at the end of the paper. Two reasons exist for this. First, to show that it is possible to analyze not only the optimize behaviour banks but also debtors. Moreover, the theory of stochastic programming can be employed to it. However to present a suitable numerical solution is beyond of the scop of this paper. Maybe, it appears later including more complicated model.

\section{ACKNOWLEDGEMENT}

This work was supported by the Czech Science Foundation under Grants 15-10331S. 
[1] L.Dai, C.H. Chen, and J.R. Birge: Convergence properties of two-stage stochastic programming. J. Optim. Theory Appl. 106 (2000), 489-509. DOI:10.1023/a:1004649211111

[2] J. Dupačová and R. J.-B. Wets: Asymptotic behaviour of statistical estimates and optimal solutions of stochastic optimization problems. Ann. Statist. 16 (1984), 1517-1549. DOI:10.1214/aos/1176351052

[3] J. Dupačová, J. Hurt, and J. Štěpán: Stochastic Modelling in Economics and Finance. Kluwer, Dordrecht 2002.

[4] W. Hoeffding: Probability inequalities for sums of bounded random variables. J. Amer. Statist. Assoc. 58 (1963), 301, 13-30. DOI:10.1080/01621459.1963.10500830

[5] M. Houda and V. Kaňková: Empirical estimates in economic and financial optimization problems. Bull. Czech Econometr. Soc. 19 (2012), 29, 50-69.

[6] Y. M. Kaniovski, A. J. King, and R. J.-B. Wets: Probabilistic bounds (via large deviations) for the solutions of stochastic programming problems. Ann. Oper. Res. 56 (1995), 189-208. DOI:10.1007/bf02031707

[7] V. Kaňková: Optimum solution of a stochastic optimization problem with unknown parameters. In: Trans. 7th. Prague Conf. 1974, Academia, Prague 1977, pp. 239-244.

[8] V. Kaňková: An approximative solution of stochastic optimization problem. In: Trans. 8th. Prague Conference, Academia, Prague 1978, pp. 349-353. DOI:10.1007/978-94-0099857-5_33

[9] V. Kaňková: Uncertainty in stochastic programming. In: Proc. Inter. Conf. on Stoch. Optim., Kiev 1984 (V.I Arkin and R. J.-B. Wets, eds.), Lecture Notes in Control and Information Sciences 81, Springer, Berlin 1986, pp. 393-401. DOI:10.1007/bfb0007116

[10] V. Kaňková: On the stability in stochastic programming: the case of individual probability constraints. Kybernetika 33 (1997), 5, 525-546.

[11] V. Kaňková and M. Houda: Empirical estimates in stochastic programming. In: Proc. Prague Stochastics 2006 (M. Hušková and M. Janžura, eds.), MATFYZPRESS, Prague 2006, pp. 426-436.

[12] V. Kaňková: Multistage stochastic programs via autoregressive sequences and individual probability constraints. Kybernetika 44 (2008), 2, 151-170.

[13] V. Kaňková: Empirical estimates in optimization problems; survey with special regard to heavy tails and dependent samples. Bull.Czech Econometric. soc. 19 (2012), 30, 92-111.

[14] V. Kaňková and M. Houda: Thin and heavy tails in stochastic programming. Kybernetika 51 (2015), 3, 433-456. DOI:10.14736/kyb-2015-3-0433

[15] V. Kaňková: Scenario generation via $\mathcal{L}_{1}$ norm. In: Proc. 33rd Inter. Conf. Mathematical Methods in Economics 2015 (D. Marinčík, J. Ircingová and P. Janeček, eds.), Published by West Bohemia, Plzeñ 2015, pp. 331-336.

[16] V. Kaňková: A note on optimal value of loans. In: Proc. 34th Inter. Conf. Mathematical methods in economics 2016 (A. Kocourek and M. Vavroušek, eds.), Technical University Liberec, Liberec 2016, pp, 371-376.

[17] B. Luderer, V. Nollau, and K. Vetters: Mathematical Formulas for Economists. Third edition. Springer Science and Media, 2006. DOI:10.1007/978-3-662-12431-4

[18] G. Ch. Pflug: Scenarion tree generation for multiperiod finncial optimization by optimal discretizatin. Math. Program. Ser. B 89 (2001), 251-271. DOI:10.1007/pl00011398 
[19] G. Ch. Pflug: Stochastic Optimization and Statistical Inference. In: Stochastic Programming, Handbooks in Operations Research and Managemennt Science, Vol. 10 (A. Ruszczynski and A.A. Shapiro, eds.), Elsevier, Amsterdam 2003, pp. 427-480. DOI:10.1016/s0927-0507(03)10007-2

[20] R. Rockafellar and R. J. B. Wets: Variational Analysis. Springer, Berlin 1983. DOI:10.1007/978-3-642-02431-3

[21] W. Römisch and R. Schulz: Stability of solutions for stochastic programs with complete recourse. Math. Oper. Res. 18 (1993), 590-609. DOI:10.1287/moor.18.3.590

[22] W. Römisch: Stability of Stochastic Programming Problems. In: Stochastic Programming, Handbooks in Operations Research and Managemennt Science, Vol. 10 (A. Ruszczynski and A. A. Shapiro, eds.), Elsevier, Amsterdam 2003, pp. 483-554. DOI:10.1016/s09270507(03)10008-4

[23] G. Salinetti and R. J. B. Wets: On the convergence of closed-valued measurable multifunctions. Trans. Amer. Math. Society 266 (1981), 1, 275-289. DOI:10.1090/s0002-99471981-0613796-3

[24] R. Schulz: Rates of convergence in stochastic programs with complete integer recourse. SIAM J. Optim. 6 (1996), 4, 1138-1152. DOI:10.1137/s1052623494271655

[25] A. Shapiro: Quantitative stability in stochastic programming. Math. Program. 67 (1994), 99-108. DOI:10.1007/bf01582215

[26] A. Shapiro, D. Dentcheva, and A. Ruszczynski: Lectures on Stochastic Programming (Modeling and Theory). Published by Society for Industrial and Applied Mathematics and Mathematical Programming Society, Philadelphia 2009. DOI:10.1137/1.9780898718751

[27] M. Śmíd: The expected loss in the discretization of multistage stochastic programming problems-estimation and convergence rate. Ann. Oper. Res. 165 (2009), 29-45. DOI:10.1007/s10479-008-0355-9

[28] M. Šmíd and J. Dufek: Multi-period Factor Model of Loan Portfolio (July 10, 2016). DOI:10.2139/ssrn.2703884

[29] G. R. Shorack and J. A. Wellner: Empirical Processes and Applications to Statistics. Wiley, New York 1986. DOI:10.1137/1.9780898719017

[30] R. J. B. Wets: A Statistical Approach to the Solution of Stochastic Programs with (Convex) Simple Recourse. Research Report, University Kentucky 1974.

Vlasta Kaňková, Institute of Information Theory and Automation, The Czech Academy of Sciences, Pod Vodárenskou věži 4, 18208 Praha 8. Czech Republic.

e-mail: kankova@utia.cas.cz 